\title{
Parameters for Fabricating Nano-Au Colloids through the Electric Spark Discharge Method with Micro-Electrical Discharge Machining
}

\author{
Kuo-Hsiung Tseng *, Meng-Yun Chung and Chaur-Yang Chang \\ Department of Electrical Engineering, National Taipei University of Technology, Taipei 10608, Taiwan; \\ avicwizard@hotmail.com (M.-Y.C.); cychang@ntut.edu.tw (C.-Y.C.) \\ * Correspondence: khtseng@ee.ntut.edu.tw; Tel.: +886-2771-2171 (ext. 2173)
}

Academic Editor: Thomas Nann

Received: 2 April 2017; Accepted: 30 May 2017; Published: 2 June 2017

\begin{abstract}
In this study, the Electric Spark Discharge Method (ESDM) was employed with micro-electrical discharge machining (m-EDM) to create an electric arc that melted two electrodes in deionized water (DW) and fabricated nano-Au colloids through pulse discharges with a controlled on-off duration $\left(\mathrm{T}_{\mathrm{ON}}-\mathrm{T}_{\mathrm{OFF}}\right)$ and a total fabrication time of $1 \mathrm{~min}$. A total of six on-off settings were tested under normal experimental conditions and without the addition of any chemical substances. Ultraviolet-visible spectroscopy (UV-Vis), Zetasizer Nano measurements, and scanning electron microscopy-energy dispersive X-ray (SEM-EDX) analyses suggested that the nano-Au colloid fabricated at 10-10 $\mu \mathrm{s}$ ( $10 \mu \mathrm{s}$ on, $10 \mu \mathrm{s}$ off) had higher concentration and suspension stability than products made at other $\mathrm{T}_{\mathrm{ON}}-\mathrm{T}_{\mathrm{OFF}}$ settings. The surface plasmon resonance (SPR) of the colloid was $549 \mathrm{~nm}$ on the first day of fabrication and stabilized at $532 \mathrm{~nm}$ on the third day. As the $\mathrm{T}_{\mathrm{ON}}-\mathrm{T}_{\mathrm{OFF}}$ period increased, the absorbance (i.e., concentration) of all nano-Au colloids decreased. Absorbance was highest at $10-10 \mu \mathrm{s}$. The SPR peaks stabilized at $532 \mathrm{~nm}$ across all $\mathrm{T}_{\mathrm{ON}}-\mathrm{T}_{\mathrm{OFF}}$ periods. The Zeta potential at 10-10 $\mu$ s was $-36.6 \mathrm{mV}$, indicating that no nano-Au agglomeration occurred and that the particles had high suspension stability.
\end{abstract}

Keywords: electric spark discharge method; micro-electrical discharge machining; nano-Au colloid; nanosuspension stability

\section{Introduction}

Across the world and throughout history, gold has been regarded as a symbol of nobility. Its glittering beauty attracts the eye. Gold is a highly stable element used for decoration and added as an antioxidant to tea, wine, and certain foods [1]. However, at the nanoscale, gold turns dark because it absorbs the light of certain wavelengths [2]. In addition to being medically exploited for its antioxidant effects, nano-Au has a great potential for use in DNA chips designed to detect hereditary diseases; thus, its fabrication has been extensively studied [3].

Currently, the preparation methods for nanometal colloids are primarily divided into physical and chemical methods. However, the typical chemical methods require adding some chemical agents, so the products contain other derivatives [4]. Moreover, nanometal colloids prepared through chemical methods are easily contaminated during preparation. Human beings can be harmed if they are exposed to nanoscale particles that cause ailments of the lungs and other organs [5,6]. Therefore, to solve the problem of dust drift in the processing environment is a major challenge of nanotechnology research.

Several studies have discussed the application of Electrical Discharge Machining (EDM) in nanomanufacturing [7-9]. The experimental conditions for implementing EDM in nanomanufacturing are described as follows: (1) one electrode is attached to the bottom of a vessel with a direct current (DC) 
power source, and deionized water (DW) is subsequently added to the vessel [10]; (2) another electrode is attached to a servomechanism, which adjusts the gap between the two electrodes; (3) the on-off duration of pulse discharge $\left(\mathrm{T}_{\mathrm{ON}}-\mathrm{T}_{\mathrm{OFF}}\right)$, current intensity, and other parameters are specified on a control panel [11]; and (4) a magnetic stirrer is used to rotate a magnet in the vessel, thereby stirring the DW in the vessel and distributing nanoparticles evenly in the liquid. Nano-Au colloids prepared through EDM have exhibited absorbance spectra with surface plasmon resonance (SPR) peaks at 544 $\mathrm{nm}$ and a zeta potential at $-40 \mathrm{mV}$, as observed through ultraviolet-visible spectroscopy (UV-Vis) and Zetasizer Nano measurement. However, fabricating nano-Au colloids through EDM entails considerable costs, and the fabrication process cannot be monitored in real time. Furthermore, wear on the machine's components due to long-term use undermines the efficiency of the process. The efficiency of fabricating nanometal colloids is proportional to the discharge success rate. Observing the discharge success rate in real time during fabrication can help to resolve malfunctions and to improve the quality of the products. For example, when the two electrodes become misaligned, the success rate declines, but that success rate can be regained by realigning the electrodes in a straight line. Traditional EDM can monitor only the current of the gap between the electrodes; it cannot identify the discharge success rate. To solve this problem, the discharge success rate circuitry in this study was designed to monitor the discharge success rate in real time. To ameliorate the shortcomings of EDM [12], this study proposed a micro-Electrical Discharge Machining (m-EDM) for fabricating nano-Au; this m-EDM was found to be suitable for nanomanufacturing.

\section{Results}

\subsection{Nano-Au Colloid Preparation}

Optimal Parameters for Fabricating Nano-Au Colloids through m-EDM

Table 1 lists the parameters used for fabricating nano-Au colloids through m-EDM. At the standard temperature and pressure, a proportional-integral-derivative (PID) controller was configured with the proportional controller setting $\mathrm{Kp}=0.21$, integral controller setting $\mathrm{Ki}=0.25$, and derivative controller setting $\mathrm{Kd}=0.015 ; 99.99 \%$ pure metal electrodes with a diameter of $1 \mathrm{~mm}$ were prepared in $100 \mathrm{~mL}$ of DW at different periods of $\mathrm{T}_{\mathrm{ON}}-\mathrm{T}_{\mathrm{OFF}}$ (for a total discharge time of $1 \mathrm{~min}$ per configuration) to produce nano-Au colloids. A necessary condition of $\mathrm{m}-\mathrm{EDM}$ with high process efficiency is that the electrode gap in the discharge process must be maintained within a small range. To meet the conditions, proportional-integral-derivative (PID) control was used. Through PID control, the positive and negative rotation of the motor can be regulated to maintain the electrodes within a range of $30 \mu \mathrm{m}$.

Table 1. Parameters used for the fabrication of nano-Au colloids through micro-Electrical Discharge Machining (m-EDM).

\begin{tabular}{|c|c|c|c|c|c|}
\hline Temperature & $\begin{array}{l}\text { Deionized } \\
\text { Water }\end{array}$ & Electrode & Discharge Time & Voltage & Current \\
\hline $25^{\circ} \mathrm{C}$ & DW & $\mathrm{Au}$ & $1 \mathrm{~min}$ & $100 \mathrm{~V}$ & Approx. $6.5 \mathrm{~A}$ \\
\hline $\begin{array}{c}\text { Atmospheric } \\
\text { pressure }\end{array}$ & $\begin{array}{c}\text { Volume of the } \\
\text { beaker }\end{array}$ & $\begin{array}{l}\text { Purity of the } \\
\text { electrode }\end{array}$ & $\begin{array}{c}\text { Diameter of the } \\
\text { electrode } \\
\text { (positive/negative) }\end{array}$ & $\begin{array}{l}\text { On-off duration } \\
\text { of pulse discharge } \\
\left(\mathrm{T}_{\mathrm{ON}}-\mathrm{T}_{\mathrm{OFF}}\right)\end{array}$ & PID parameters \\
\hline \multirow{6}{*}{$1 \mathrm{~atm}$} & \multirow{6}{*}{$100 \mathrm{~mL}$} & \multirow{6}{*}{$99.99 \%$} & \multirow{6}{*}{$1 / 1 \mathrm{~mm}$} & $10-10 \mu \mathrm{s}$ & \multirow{6}{*}{$\begin{array}{c}\mathrm{Kp}=0.21 \\
\mathrm{Ki}=0.25 \\
\mathrm{Kd}=0.015\end{array}$} \\
\hline & & & & $20-20 \mu \mathrm{s}$ & \\
\hline & & & & $30-30 \mu \mathrm{s}$ & \\
\hline & & & & $40-40 \mu \mathrm{s}$ & \\
\hline & & & & $50-50 \mu \mathrm{s}$ & \\
\hline & & & & $100-100 \mu \mathrm{s}$ & \\
\hline
\end{tabular}


On the basis of daily UV-Vis analysis results (Figure 1), nano-Au colloids prepared with $\mathrm{T}_{\mathrm{ON}}-\mathrm{T}_{\mathrm{OFF}}$ values of 10-10, 20-20, 30-30, 40-40, 50-50, and 100-100 $\mu$ s had SPR peaks of 549, 531, 531, 531, 531, and $532 \mathrm{~nm}$, respectively, with corresponding absorbance values of $0.113,0.077,0.057,0.039,0.035$, and 0.016 . The nano-Au colloid prepared at a $\mathrm{T}_{\mathrm{ON}}-\mathrm{T}_{\mathrm{OFF}}$ of $10-10 \mu \mathrm{s}$ had the highest absorbance and concentration, whereas that prepared at a $\mathrm{T}_{\mathrm{ON}}-\mathrm{T}_{\mathrm{OFF}}$ of $100-100 \mu$ s had the lowest absorbance and concentration. The SPR peak was slightly higher at $10-10 \mu \mathrm{s}(549 \mathrm{~mm})$ than at other $\mathrm{T}_{\mathrm{ON}}-\mathrm{T}_{\mathrm{OFF}}$ periods.

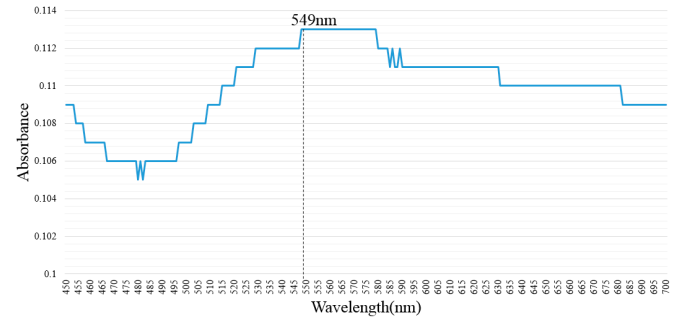

(a)

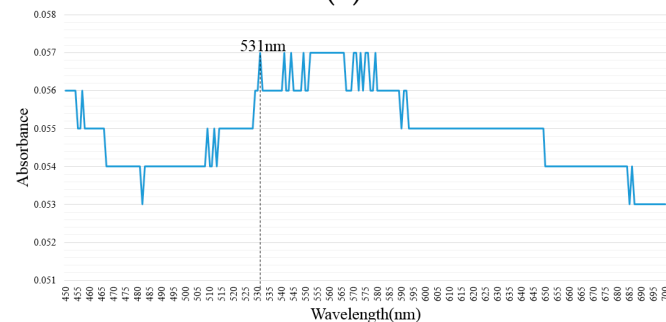

(c)

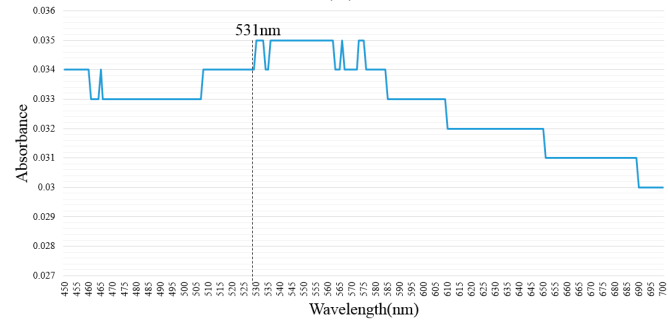

(e)

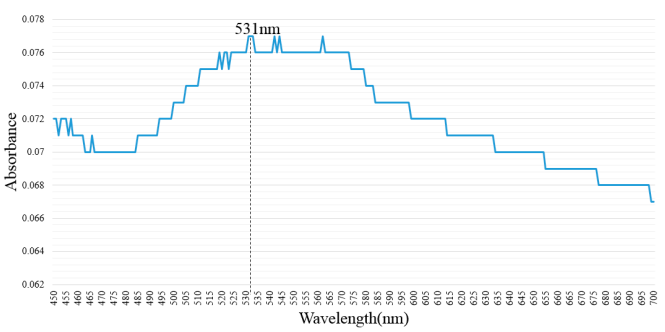

(b)

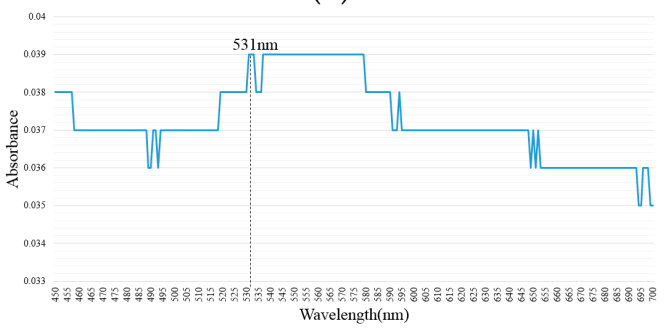

(d)

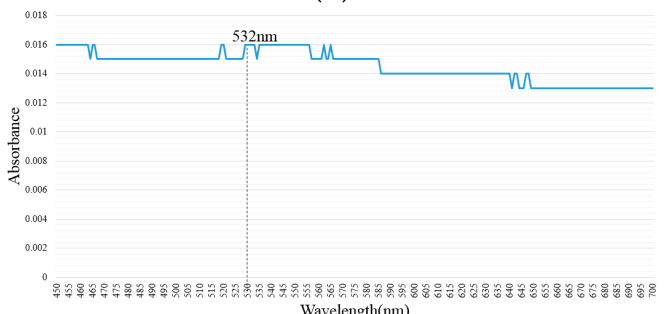

(f)

Figure 1. Daily ultraviolet-visible spectroscopy (UV-Vis) analysis results of nano-Au colloids fabricated

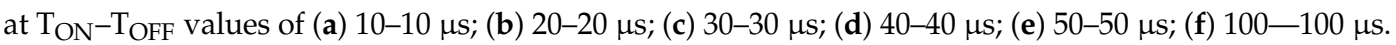

The Zeta potential was $-36.6 \mathrm{mV}$ for the nano-Au colloid prepared at 10-10 $\mu$ s (Figure 2a) and $-37.6 \mathrm{mV}$ for that prepared at 50-50 $\mu$ s (Figure $2 \mathrm{~b}$ ). An absolute Zeta potential value of over $30 \mathrm{mV}$ indicates high suspension stability in the nanoparticles. Therefore, nano-Au colloids fabricated through m-EDM exhibited high suspension stability.

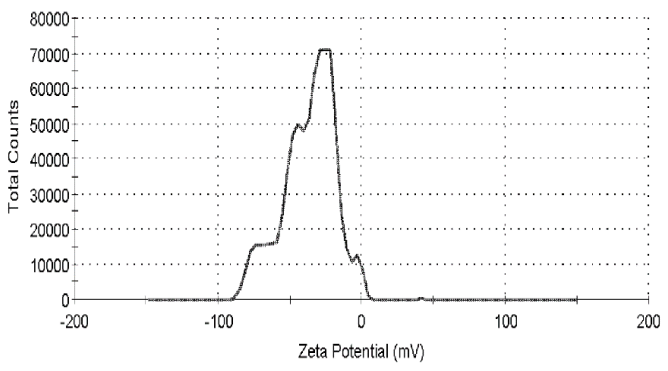

(a)

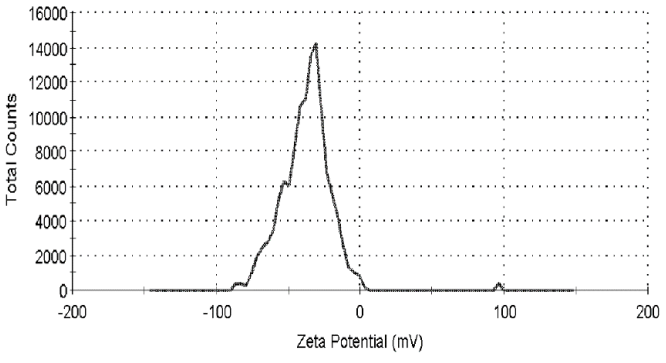

(b)

Figure 2. Daily Zeta potential analysis results of nano-Au colloids fabricated at $\mathrm{T}_{\mathrm{ON}}-\mathrm{T}_{\mathrm{OFF}}$ settings of

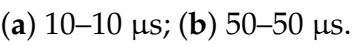


Figure 3a shows a scanning electron microscopy (SEM) image of a nano-Au colloid fabricated through m-EDM. Each grid of the image is $30 \mathrm{~nm}$; most nanoparticles are less than $30 \mathrm{~nm}$ in diameter, circular in shape, and agglomerated in several grids. Figure 3b presents the EDX results of the colloid, which indicate that the dominant element of nanoparticles fabricated through the Electric Spark Discharge Method (ESDM) is gold. This figure also shows different peaks, indicating that the colloid was not $100 \%$ pure and both carbon paste and copper gauze were used in the EDX analysis. Thus, minor elements (such as $\mathrm{Cu}, \mathrm{C}$, and $\mathrm{O}$ ) identified in the colloid analysis are measuring errors. Figure 3c illustrates the size distribution of the nano-Au particles in the colloid. It indicates that most of the nano-Au particles fabricated by m-EDM are smaller than $100 \mathrm{~nm}$. They are all nanoscale particles.

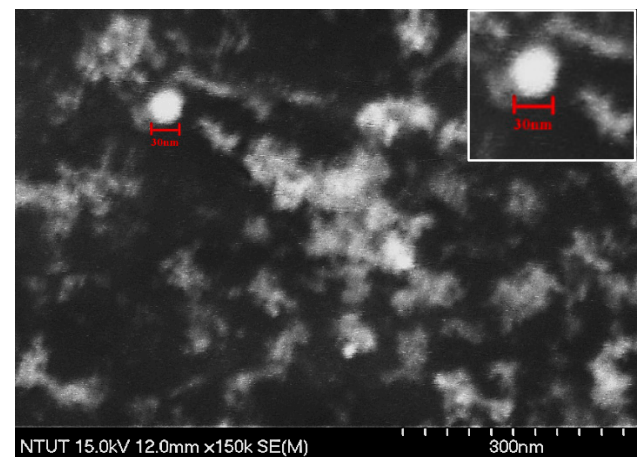

(a)

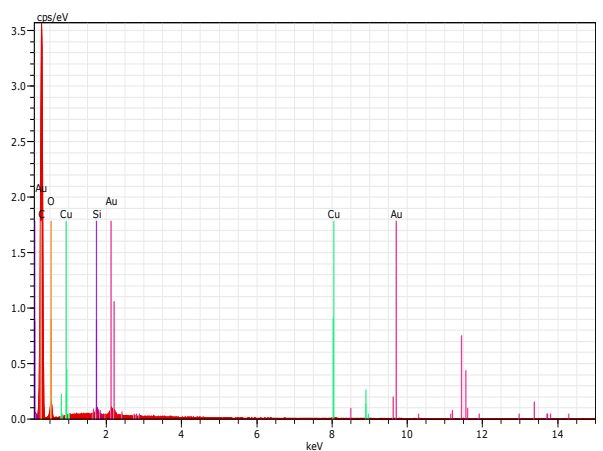

(b)

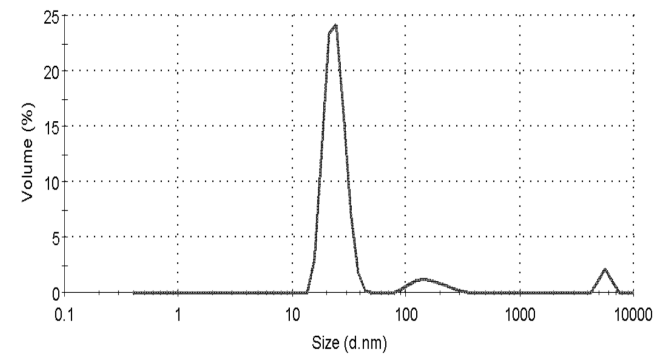

(c)

Figure 3. (a) Scanning electron microscopy (SEM) image; (b) energy dispersive X-ray (EDX) analyses of the elements of a nano-Au colloid; (c) size distribution in volume of nano-Au particles in the colloid.

\subsection{Suspension Analysis of Nano-Au Colloids Fabricated through m-EDM}

Figure 4 compares the SPR peaks and absorbance from a 5-day UV-Vis observation of nano-Au colloids fabricated at different $\mathrm{T}_{\mathrm{ON}}-\mathrm{T}_{\mathrm{OFF}}$ settings. The square dots indicate the SPR peaks, whereas the circular dots denote absorbance. From the third day of observation across all $\mathrm{T}_{\mathrm{ON}}-\mathrm{T}_{\mathrm{OFF}}$ periods, the SPR peaks began to stabilize, whereas absorbance decreased consistently because some of the colloids continued to agglomerate and precipitate, causing their concentrations to decrease.

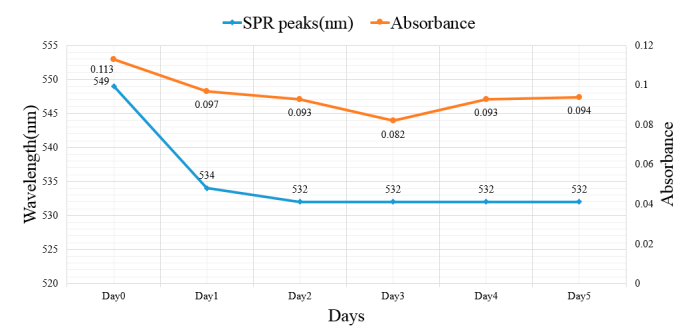

(a)

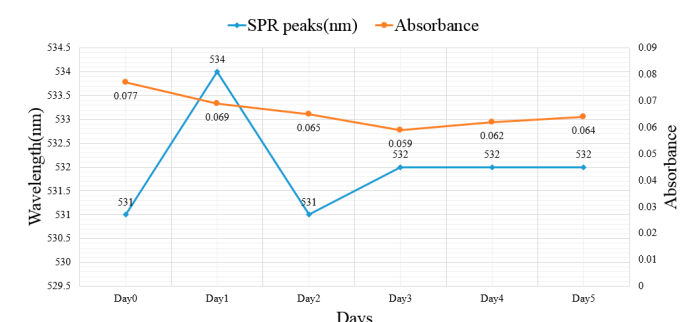

(b)

Figure 4. Cont. 


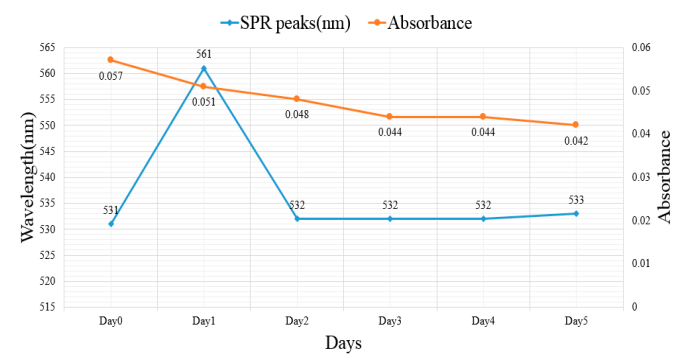

(c)

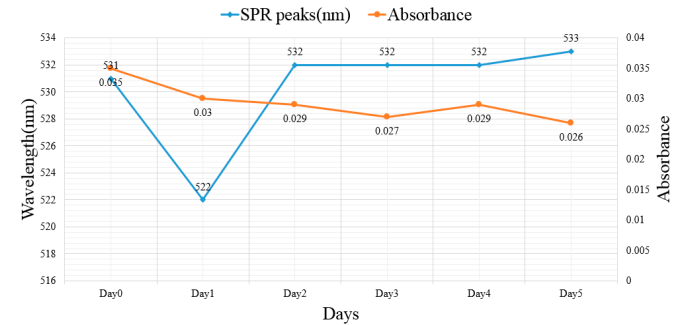

(e)

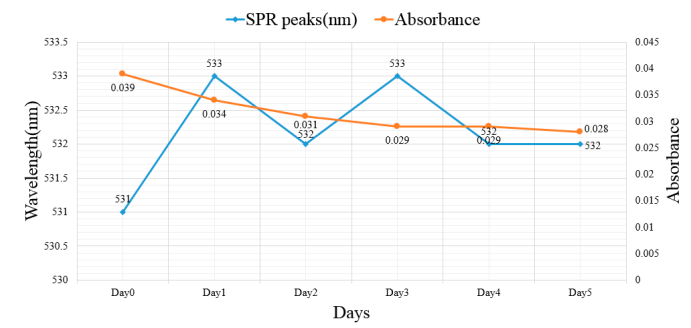

(d)

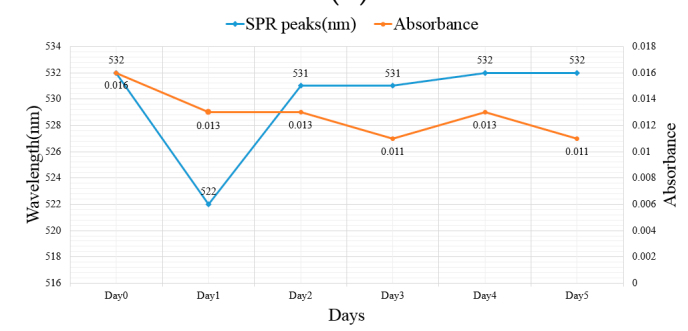

(f)

Figure 4. Results of an UV-Vis observation of the surface plasmon resonance (SPR) peaks and absorbance of nano-Au colloids fabricated at $\mathrm{T}_{\mathrm{ON}}-\mathrm{T}_{\mathrm{OFF}}$ settings of (a) 10-10 $\mu \mathrm{s}$; (b) 20-20 $\mu \mathrm{s}$;

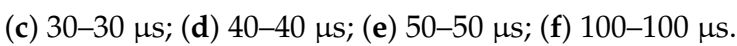

\section{Discussion}

In summary, this study used $\mathrm{m}$-EDM to fabricate nano-Au colloids at different $\mathrm{T}_{\mathrm{ON}}-\mathrm{T}_{\mathrm{OFF}}$ settings with a total fabrication time of $1 \mathrm{~min}$ for each configuration. The SPR of the nano-Au colloid fabricated at the $\mathrm{T}_{\mathrm{ON}}-\mathrm{T}_{\mathrm{OFF}}$ of 10-10 $\mathrm{\mu s}$ was $549 \mathrm{~nm}$ on the first day of fabrication; it stabilized at $532 \mathrm{~nm}$ and exhibited a red shift (which indicated the shrinking of nano-Au particles) on the third day. In general, the peaks of nanoscale materials exhibit displacement, which is characterized by either a red or blue shift. A red shift means the wavelength is moving in the dominant direction and thus indicates that nanoparticles are agglomerating increasing in size. A blue shift indicates that nanoparticles are decreasing in size. Moreover, the absorbance of colloids varies depending on their concentrations: colloids with high concentrations, which are characterized by dense particle distributions, have high absorbance; whereas those with low concentrations have low absorbance. Accordingly, the nano-Au particles were either precipitated or dispersed in DW several days after they were prepared. Therefore, across all $\mathrm{T}_{\mathrm{ON}}-\mathrm{T}_{\mathrm{OFF}}$ settings, absorbance (i.e., concentration) correlated negatively with $\mathrm{T}_{\mathrm{ON}}-\mathrm{T}_{\mathrm{OFF}}$; absorbance peaked at 10-10 $\mu$ s.

The SPRs of all nano-Au colloids stabilized at $532 \mathrm{~nm}$, regardless of the $\mathrm{T}_{\mathrm{ON}}-\mathrm{T}_{\mathrm{OFF}}$ setting. The Zeta potential at $10-10 \mu$ s was $-36.6 \mathrm{mV}$, suggesting high suspension stability in nano-Au colloids fabricated at this $\mathrm{T}_{\mathrm{ON}}-\mathrm{T}_{\mathrm{OFF}}$ setting; the nanoparticles of those colloids did not agglomerate. Table 2 presents a comprehensive comparison of all nano-Au colloids fabricated through m-EDM. SEM revealed that the particles in the nano-Au colloids were spherical in shape and less than $100 \mathrm{~nm}$ in size.

Table 2. Comprehensive comparison of all nano-Au colloids fabricated through m-EDM.

\begin{tabular}{ccccc}
\hline Days & \multicolumn{2}{c}{ Day 0 } & \multicolumn{2}{c}{ Day 5 } \\
\hline T $_{\text {ON }}-\mathbf{T}_{\text {OFF }}$ & SPR Peaks (nm) & Absorbance & SPR Peaks (nm) & Absorbance \\
\hline $10-10 \mu \mathrm{s}$ & 549 & 0.113 & 532 & 0.094 \\
$20-20 \mu \mathrm{s}$ & 531 & 0.077 & 532 & 0.064 \\
$30-30 \mu \mathrm{s}$ & 531 & 0.057 & 533 & 0.042 \\
$40-40 \mu \mathrm{s}$ & 531 & 0.039 & 532 & 0.028 \\
$50-50 \mu \mathrm{s}$ & 531 & 0.035 & 533 & 0.026 \\
$100-100 \mu \mathrm{s}$ & 532 & 0.016 & 532 & 0.011 \\
\hline
\end{tabular}




\section{Materials and Methods}

\subsection{Fabricating Nano-Au Colloids through the Electric Spark Discharge Method (ESDM)}

The proposed ESDM involves preparing nano-Au colloids in a simple, convenient, and low-cost manner, without the addition of any materials, and is an environmentally friendly nanophysical fabrication method for such colloids because, unlike costly vacuum EDM methods, it produces no waste [13].

The ESDM delivers DC voltages of tens to hundreds of volts between two metal electrodes, and uses a servomechanism to control the cathode, allowing the electrode to inch toward the anode. Thus, when the distance between the two electrodes narrows to approximately $30 \mu \mathrm{m}$, the strength of the electric field located between two salient points on the electrode surface surpasses the dielectric strength of insulating liquids around the electrodes, leading to insulation breakdown in the liquids (Figure 5a). A discharge column appears between the electrodes and an electric discharge begins in the same position (Figure 5b) [14]. At a high arc temperature, the electrodes melt or even vaporize, ejecting their melted particles and allowing them to cool rapidly, freeze, and form nanoparticles in insulating liquid (Figure 5c). When the electric discharge ends, the discharge column disappears, eliminating nanoparticles, and DW resumes insulation (Figure 5d). This process is performed repeatedly to yield nano-Au colloids. In summary, the ESDM uses arc discharges to melt the surfaces of the anode and cathode, thereby yielding nano-Au colloids [15].

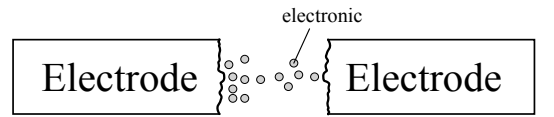

(a)

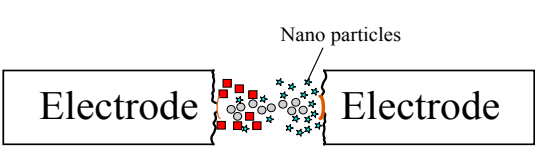

(c)

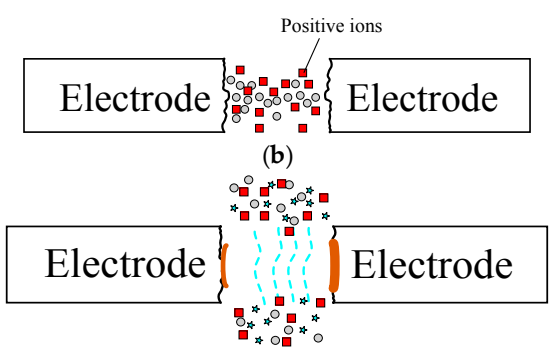

(d)

Figure 5. Illustration of the Electric Spark Discharge Method (ESDM). (a) Electrode surface surpasses the dielectric strength; (b) A discharge column appears; (c) The electrodes melt or even vaporize, ejecting their melted particles; (d) DW resumes insulation.

Figure 6 depicts the procedure used for the fabrication of nano-Au colloids through the ESDM. First, two selected metal electrodes are rinsed with a proper volume of DW and attached in a beaker to connect to the apparatus. Second, after fabrication parameters (e.g., $\mathrm{T}_{\mathrm{ON}}-\mathrm{T}_{\mathrm{OFF}}$ ) are specified and the electric discharge time is adjusted according to the type of the metal, fabrication begins. Nano-Au colloids are obtained following the electric discharge. Finally, the colloids are examined through UV-Vis, a Zetasizer, and SEM-EDX [16].

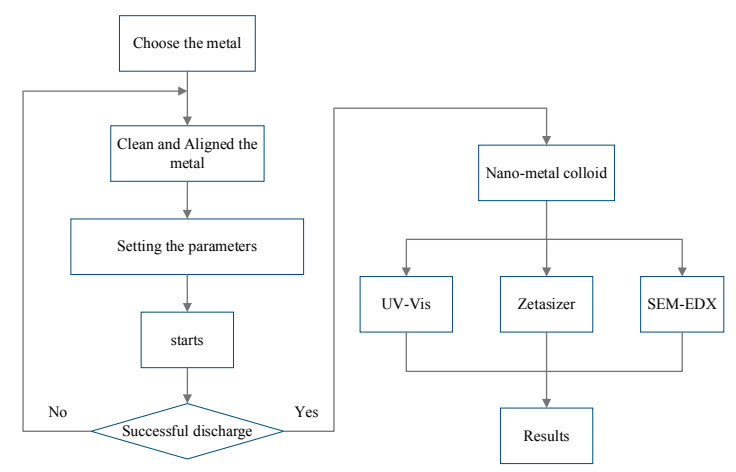

Figure 6. Procedure for fabricating nano-Au colloids through the ESDM. 


\subsection{Micro-Electrical Discharge Machine}

In this study, micro-electrical discharge machining (m-EDM) was used to ensure the continuity, reproducibility, and integrity of the processes and to optimize fabrication conditions. The duration of $\mathrm{T}_{\mathrm{ON}}-\mathrm{T}_{\mathrm{OFF}}$, the electricity consumption of the electrodes, the completion time, and the PID parameters can be specified in m-EDM, and the subsystems can be optimized [17]. An m-EDM apparatus is inexpensive, small, and easy to operate and maintain, and fabricates nano-Au colloids efficiently. The apparatus in this study comprised five subsystems: (1) computer operating system; (2) I/O insulation circuit board; (3) circuitry system; (4) power supply system; and (5) experimental system (Figure 7). Each of these systems is detailed in the following passages.

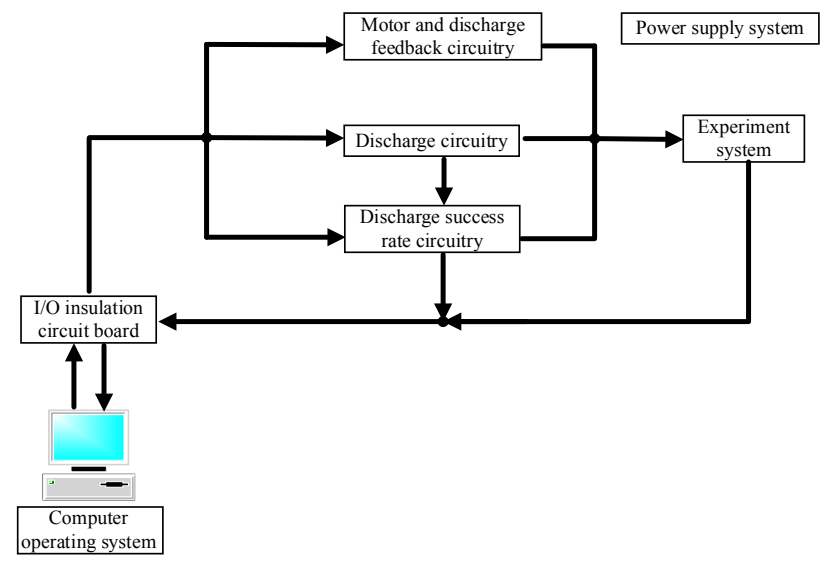

Figure 7. Configuration of m-EDM.

Computer operating system. The computer operating system uses VisSim to determine the start and finish time, PID parameters, and $\mathrm{T}_{\mathrm{ON}}-\mathrm{T}_{\mathrm{OFF}}$ of $\mathrm{m}-\mathrm{EDM}$. VisSim is a software program for the simulation and real-time control of dynamic systems. It efficiently and accurately executes various simulations-both linear and nonlinear, continuous and discrete, time-dependent and non-time-dependent. The program provides an integrated environment for system design. Its visual interface eliminates the need for manually written code. The VisSim environment in this study included a built-in RT/DAC4 board (a hybrid I/O platform with a personal computer interface (PCI) card) that used multiple digital and analog I/O modules to obtain data and control dynamic systems in real time. The RT/DAC4 board contained a field-programmable gate array chip responsible for computing digital signals.

I/O insulation circuit board. The name of the board was DB-8025. Because VisSim was installed on the host computer, the board separated the circuitry area from the host to prevent the host from being damaged by pulsed voltages or currents.

Circuitry system. This system comprised three subsystems: discharge circuitry, motor and discharge feedback circuitry, and discharge success rate circuitry. The details of these three items were as follows.

Discharge circuitry. This circuitry connected two $50 \mathrm{~V}$ DC power supplies in series to supply a voltage of $100 \mathrm{~V}$ and a current of $2.3 \mathrm{~A}$. It used transistors to control the switch of the discharge loop. The discharge frequency of the circuitry was $0-50 \mathrm{kHz}$. $\mathrm{T}_{\mathrm{ON}}-\mathrm{T}_{\mathrm{OFF}}$ was specified through the computer. A light-emitting diode turned on when any electric discharge occurred and turned off when the discharge had finished. Pulse-width modulation (PWM) signals were supplied by the gates of IRF740 power field-effect transistors, which were in the conducting state at high potentials or in the cutoff state at low potentials. PWM signals were generated by VisSim; they were output by a motion control interface card and delivered through an optically coupled 6N137 guard circuit to the gates of the transistors. 
Motor and discharge feedback circuitry. This circuitry comprised a discharge loop circuit and motor circuit. The discharge loop circuit captured the voltage in the gap between two electrodes (Vgap), processed signals, and sent them back to the computer as feedback signals to control the motor. The signal of Vgap was delivered by a differential amplifier into a low-pass filter, thereby converting PWM signals into analog signals at a time constant of $1 \mathrm{~ms}$ (therefore, the analog signals had to be at least 10 times stronger than the cycle of discharge). The low-pass filter can be perceived as an integrator; thus, a PWM signal varied along with its analog level [18]. Analog signals converted from PWM signals were subsequently delivered through the I/O insulation circuit board to the computer. In the motor circuit, VisSim generated a voltage of -2.4 to $2.4 \mathrm{~V}$ to determine the sliding movement of the motor (i.e., going forward or backward) by the voltages $\mathrm{Vg}$ (which was within a specific range of voltage) and Vgap during discharge success. The gap between the two electrodes was adjusted in three different ways (Figure 8): (a) when both electrodes were in an open circuit and Vgap was at its highest level (higher than $\mathrm{Vg}$ ), the gap between the electrodes became excessively wide and VisSim supplied a voltage of 0 to $2.4 \mathrm{~V}$ to the motor, allowing it to advance and slide forward; (b) when both electrodes discharged electricity successfully, Vgap decreased to the level of $\mathrm{Vg}$; the gap between electrodes was 10-30 $\mu \mathrm{m}$, and the motor remained motionless; and (c) when both electrodes were in short circuit and Vgap was zero, VisSim supplied a voltage of -2.4 to $0 \mathrm{~V}$ and the motor applied a force to slide the sliding table backward. This circuitry included a motor driver with a power-source voltage of $24 \mathrm{~V}$ and PWM as the output mode; the motor driver adjusted the PWM duty cycle in line with its voltage reference (REF+), which was directly proportional to the revolutions per minute of the motor. Electric-potential commands were delivered through an analog isolation circuit into the REF+ pin of the driver.

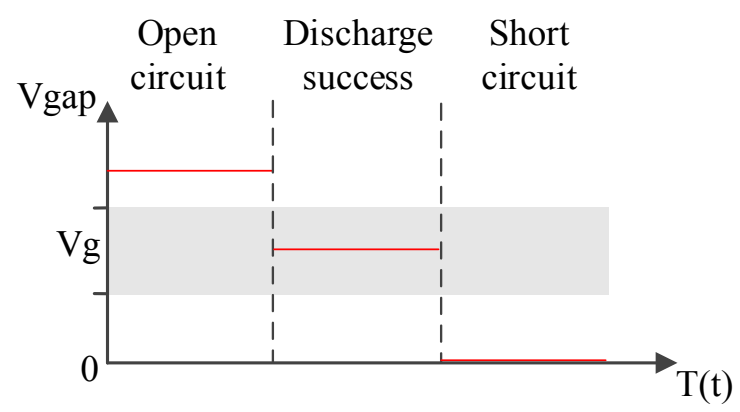

Figure 8. Adjusting the gap between the two electrodes in three different ways.

Discharge success rate circuitry. The differential amplifier captured Vgap and Igap as the output signals for a transistor-transistor logic circuit. The circuit determined the signals Vgap and Igap, which bypassed a digital isolation circuit and formed an AND gate with a $1 \mathrm{MHz}$ pulse wave to output a pulse-wave signal to the counter pin. Afterwards, VisSim determined the number of successful electric discharges by the number of occurrences of the pulse wave and presented its estimates on a computer screen, which provided an overview of the electric discharges during nano-Au colloid fabrication.

Power supply system. This system comprised three circuit boards and a motor power supply.

Experiment system. This horizontally structured (Figure 9) system enabled easy operation. Vertical electrodes have curved shapes that present difficulties. The system in this study used horizontal electrodes because they were more useful than vertical ones. As Figure 9 shows, the left electrode was fixed by a jig to the support stand and the right was fixed to the sliding table. Because of its sheer size, the magnetic stirrer was replaced by a magnet-equipped fan that distributed nanoparticles evenly in the DW. A lifting table was placed on the lifting platform, which was raised during fabrication and lowered after fabrication. The support stand was designed exclusively for the lift platform. The two electrodes were attached to a pulse-wave power supply to discharge electricity and produce nano-Au particles, which were collected in the vessel. Powered by a DC motor, the right electrode moved up or 
down the sliding track (shown on the right side of Figure 9); the position of the electrode on the track was identified by an optical encoder.

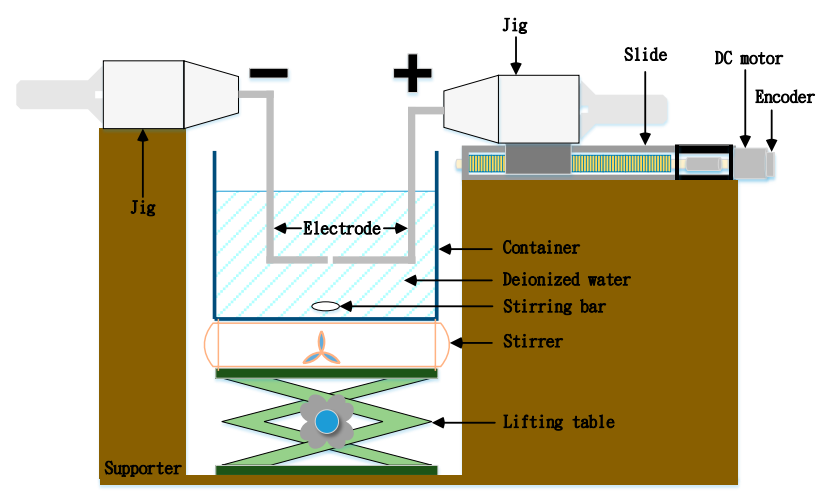

Figure 9. Structure of the electrodes of m-EDM.

\subsection{Properties of Nano-Au Colloids}

The absorbance and SPR of nano-Au colloids were determined through UV-Vis, Zeta potential and particle distribution through a Zetasizer (Malvern Zetasizer, Nano-ZS90, Worcestershire, UK), particle shapes and sizes through SEM (HITACHI, S-4700, Tokyo, Japan), and the constituent elements through X-ray (HITACHI, S-4700, Tokyo, Japan) [19].

The suspension stability of nano-Au colloids can be determined by their Zeta potential [20,21]. A high Zeta potential indicates an even distribution of nanoparticles in DW and high suspension stability, whereas a low Zeta potential suggests low suspension stability and causes nanoparticles to collide with each other and precipitate. In addition, because like electric charges repel each other, a Zeta potential with an absolute value of $30 \mathrm{mV}$ indicates high suspension stability in nanoparticles [22].

\section{Conclusions}

In this study, the ESDM was employed with m-EDM to fabricate nano-Au colloids in DW at different $\mathrm{T}_{\mathrm{ON}}-\mathrm{T}_{\mathrm{OFF}}$ settings; the surfaces of two electrodes were melted by electric arcs, under normal conditions, and without the addition of any chemical substances. The contributions of this study are listed as follows.

ESDM can be used to fabricate nano-Au colloids without the addition of any chemical substances and under normal conditions. If applied in a medical settings, such colloids would cause no harm to the human body.

The UV-Vis, Zetasizer, and SEM-EDX analyses of the nano-Au colloids produced at different $\mathrm{T}_{\mathrm{ON}}-\mathrm{T}_{\mathrm{OFF}}$ settings (as determined through VisSim) confirmed the feasibility of fabricating these colloids and their nanoparticles through $\mathrm{m}$-EDM using the ESDM.

Nano- $\mathrm{Au}$ colloids fabricated at the $\mathrm{T}_{\mathrm{ON}}-\mathrm{T}_{\mathrm{OFF}}$ setting of $10-10 \mu \mathrm{s}$ through the low-cost, easy-to-maintain, and self-designed $\mathrm{m}$-EDM had a higher suspension stability and concentration than those of colloids fabricated with any other $\mathrm{T}_{\mathrm{ON}}-\mathrm{T}_{\mathrm{OFF}}$ setting.

The wavelength of the SPRs of nano-Au colloids fabricated at different $\mathrm{T}_{\mathrm{ON}}-\mathrm{T}_{\mathrm{OFF}}$ settings stabilized at $532 \mathrm{~nm}$, indicating no agglomeration or precipitation of nanoparticles.

$\mathrm{m}$-EDM has the function of real-time monitoring, which can diagnose the problem when fabricating and improve the efficiency of m-EDM.

Acknowledgments: The authors would like to thank the Ministry of Science and Technology (NSC 103-2221-E-027-070) for financial supporting this research.

Author Contributions: Kuo-Hsiung Tseng and Meng-Yun Chung conceived and designed the experiments; Meng-Yun Chung and Chaur-Yang Chang performed the experiments; Meng-Yun Chung and Chaur-Yang Chang 
analyzed the data; Kuo-Hsiung Tseng contributed reagents/materials/analysis tools; Meng-Yun Chung wrote the paper.

Conflicts of Interest: The authors declare no conflict of interest. The founding sponsors had no role in the design of the study; in the collection, analyses, or interpretation of data; in the writing of the manuscript; or in the decision to publish the results.

\section{References}

1. Begum, N.A.; Mondal, S.; Basu, S.; Laskar, R.A.; Mandal, D. Biogenic synthesis of Au and Ag nanoparticles using aqueous solutions of Black Tea leaf extracts. Colloids Surf. B Biointerfaces 2009, 71, 113-118. [CrossRef] [PubMed]

2. Coowar, F.A.; Vitins, G.; Mepsted, G.O.; Waring, S.C.; Horsfall, J.A. Electrochemical oxidation of borohydride at nano-gold-based electrodes: Application in direct borohydride fuel cells. J. Power Sources 2008, 175, 317-324. [CrossRef]

3. Fritzsche, W.; Taton, T.A. Metal nanoparticles as labels for heterogeneous, chip-based DNA detection. Nanotechnology 2003, 14, R63-R73. [CrossRef] [PubMed]

4. Kamiar, A.; Ghotalou, R.; Valizadeh, H. Preparation, physicochemical characterization and performance evaluation of gold nanoparticles in radiotherapy. Adv. Pharm. Bull. 2013, 3, 425-428. [PubMed]

5. Ray, P.C.; Yu, H.; Fu, P.P. Toxicity and environmental risks of nanomaterials: Challenges and future needs. J. Environ. Sci. Health Part C 2009, 27, 1-35. [CrossRef] [PubMed]

6. Nowack, B.; Bucheli, T.D. Occurrence, behavior and effects of nanoparticles in the environment. Environ. Pollut. 2007, 150, 5-22. [CrossRef] [PubMed]

7. Tseng, K.-H.; Liao, C.-Y.; Huang, J.-C.; Tien, D.-C.; Tsung, T.-T. Characterization of gold nanoparticles in organic or inorganic medium (ethanol/water) fabricated by spark discharge method. Mater. Lett. 2008, 62, 3341-3344. [CrossRef]

8. Lung, J.-K.; Huang, J.-C.; Tien, D.-C.; Liao, C.-Y.; Tseng, K.-H.; Tsung, T.-T.; Kao, W.-S.; Tsaic, T.-H.; Jwo, C.-S.; Lin, H.-M.; et al. Preparation of gold nanoparticles by arc discharge in water. J. Alloys Compd. 2007, 434, 655-658. [CrossRef]

9. Tien, D.-C.; Liao, C.-Y.; Huang, J.-C.; Tseng, K.-H.; Lung, J.-K.; Tsung, T.-T.; Kao, W.-S.; Tsai, T.-H.; Cheng, T.-W.; Yu, B.-S.; et al. Novel technique for preparing a nano-silver water suspension by the arc-discharge method. Rev. Adv. Mater. Sci. 2008, 18, 750-756.

10. Son, S.; Lim, H.; Kumar, A.S.; Rahman, M. Influences of pulsed power condition on the machining properties in micro EDM. J. Mater. Process. Technol. 2007, 190, 73-76. [CrossRef]

11. Chen, D.C.; Jhang, J.J.; Guo, M.W. Application of Taguchi design method to optimize the electrical discharge machining. J. Achiev. Mater. Manuf. Eng. 2013, 57, 76-82.

12. Tseng, K.-H.; Huang, J.-C. Pulsed spark-discharge assisted synthesis of colloidal gold nanoparticles in ethanol. J. Nanopart. Res. 2011, 13, 2963-2972. [CrossRef]

13. Liao, C.-Y.; Tseng, K.-H.; Lin, H.-S. Preparation of metallic aluminum compound particles by submerged arc discharge method in aqueous media. Metall. Mater. Trans. B 2013, 44, 91-97. [CrossRef]

14. Gostimirovic, M.; Kovac, P.; Sekulic, M.; Skoric, B. Influence of discharge energy on machining characteristics in EDM. J. Mech. Sci. Technol. 2012, 26, 173-179. [CrossRef]

15. Tseng, K.-H.; Chang, C.-Y.; Chung, M.-Y.; Tang, Y.-L. Bacteriostatic Substrate by Conductivity Method and Electric Spark Discharge Method Combined with Electrospinning for Silver Dressing. Int. J. Polym. Sci. 2016, 2016, 1-10. [CrossRef]

16. Scholl, J.A.; García-Etxarri, A.; Koh, A.L.; Dionne, J.A. Observation of quantum tunneling between two plasmonic nanoparticles. Nano Lett. 2013, 13, 564-569. [CrossRef] [PubMed]

17. Tseng, K.-H.; Chiu, J.-L.; Lee, H.-L.; Kao, Y.-S.; Tien, D.-C. Spark parameter monitoring feedback system for preparation of nanosilver colloid in EDM. Mater. Manuf. Process. 2016, 31, 186-193. [CrossRef]

18. Rajurkar, K.P.; Wei, B.; Kozak, J.; McGeough, J.A. Modelling and monitoring interelectrode gap in pulse electrochemical machining. CIRP Ann. Manuf. Technol. 1995, 44, 177-180. [CrossRef]

19. Sönnichsen, C.; Reinhard, B.M.; Liphardt, J.; Alivisatos, A.P. A molecular ruler based on plasmon coupling of single gold and silver nanoparticles. Nat. Biotechnol. 2005, 23, 741-745. [CrossRef] [PubMed] 
20. Peng, X.-F.; Yu, X.-L.; Xia, L.-F.; Zhong, X. Influence factors on suspension stability of nanofluids. J. Zhejiang Univ. Eng. Sci. 2007, 41, 577-580.

21. Jailani, S.; Franks, G.V.; Healy, T.W. $\zeta$ potential of nanoparticle suspensions: Effect of electrolyte concentration, particle size, and volume fraction. J. Am. Ceram. Soc. 2008, 91, 1141-1147. [CrossRef]

22. Jiang, J.; Oberdörster, G.; Biswas, P. Characterization of size, surface charge, and agglomeration state of nanoparticle dispersions for toxicological studies. J. Nanopart. Res. 2009, 11, 77-89. [CrossRef]

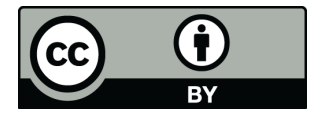

(C) 2017 by the authors. Licensee MDPI, Basel, Switzerland. This article is an open access article distributed under the terms and conditions of the Creative Commons Attribution (CC BY) license (http:/ / creativecommons.org/licenses/by/4.0/). 